\title{
A política nacional de doação de sangue pela comunidade LGBTQIA+
}

\author{
The national blood donation policy by the community LGBTQIA+ \\ La política nacional de donación de sangre de la comunidad LGBTQIA+
}

Recebido: 11/08/2021 | Revisado: 14/08/2021 | Aceito: 15/08/2021 | Publicado: 17/08/2021

\section{Resumo}

Gabriel Coutinho Gonçalves ORCID: https://orcid.org/0000-0002-5960-2976 Universidade Estadual do Ceará, Brasil E-mail: fisiogabrielcoutinho@gmail.com

Marinina Gruska Benevides ORCID: https://orcid.org/0000-0002-5656-365X Universidade Estadual do Ceará, Brasil E-mail: marininagruska@gmail.com

Rosendo Freitas de Amorim ORCID: https://orcid.org/0000-0001-6158-3826 Universidade de Fortaleza, Brasil E-mail: rosendo.freitas@gmail.com Preciliana Barreto de Morais ORCID: https://orcid.org/0000-0002-8308-9789 Universidade Estadual do Ceará, Brasil E-mail: preciliana.morais@uece.br

A exclusão sistemática de LGBTQIA+ na triagem para doação de sangue não apresenta justificativa médica absoluta, uma vez que os testes usados para seleção do sangue possuem critérios rigorosos. O presente trabalho tem por objetivo analisar a Política Nacional de Doação de Sangue no que diz respeito à população LGBTQIA+ considerando em especial o perfil de doadores de sangue em relação a comportamento de risco de contrair Infecções Sexualmente Transmissíveis quanto ao gênero e a sexualidade. Foi realizado um levantamento bibliográfico sobre a evolução de políticas públicas de doação de sangue no Brasil e no mundo entre 1970 a 2020, sendo selecionados 90 artigos científicos. Apesar da ação coercitiva do judiciário, que reconheceu que as portarias do Ministério da Saúde e da Agência Nacional de Vigilância Sanitária eram discriminatórias em relação à doações de sangue pela população nãoheterossexual, não se pode dizer que isso é condição suficiente para mitigar o preconceito e os estereótipos que recaem sobre esta população.

Palavras-chave: Doadores de sangue; Infecções sexualmente transmissíveis; Minorias sexuais e de gênero.

\begin{abstract}
The systematic exclusion of LGBTQIA+ from screening for blood donation does not have an absolute medical justification, since the tests used for blood selection have strict criteria. The present work aims to analyze the National Blood Donation Policy with regard to the LGBTQIA+ population considering in particular the profile of blood donors in relation to risk behavior of contracting Sexually Transmitted Infections regarding gender and sexuality. A bibliographical survey was carried out on the evolution of public blood donation policies in Brazil and in the world between 1970 and 2020, with 90 scientific articles selected. Despite the coercive action of the judiciary, which recognized that the ordinances of the Ministry of Health and the National Health Surveillance Agency were discriminatory in relation to blood donations by the non-heterosexual population, it cannot be said that this is a sufficient condition to mitigate prejudice and the stereotypes that befall this population.
\end{abstract}

Keywords: Blood donors; Sexually transmitted diseases; Sexual and gender minorities.

\section{Resumen}

La exclusión sistemática de LGBTQIA + en el cribado para la donación de sangre no tiene una justificación médica absoluta, ya que las pruebas utilizadas para la selección de sangre tienen criterios estrictos. El presente trabajo tiene como objetivo analizar la Política Nacional de Donación de Sangre con respecto a la población LGBTQIA + considerando en particular el perfil de los donantes de sangre en relación a las conductas de riesgo de contraer Infecciones de Transmisión Sexual en cuanto a género y sexualidad. Se realizó un relevamiento bibliográfico sobre la evolución de las políticas públicas de donación de sangre en Brasil y en el mundo entre 1970 y 2020, con 90 artículos científicos seleccionados. A pesar de la acción coercitiva del Poder Judicial, que reconoció que las ordenanzas del Ministerio de Salud y la Agencia Nacional de Vigilancia Sanitaria fueron discriminatorias en relación a las donaciones de sangre por parte de la población no heterosexual, no se puede decir que esta sea una condición suficiente para mitigar los prejuicios y los estereotipos que caen sobre esta población.

Palabras clave: Donantes de sangre; Enfermedades de transmisión sexual; Minorías sexuales y de género. 


\section{Introdução}

A politização do sangue, isto é transformar um material biológico em discussão moral, tem gerado muita controvérsia no meio acadêmico. De um lado, estão os defensores da ideia tradicional de "grupos de risco", como justificativa de exclusão de minorias historicamente perseguidas pelo estigma institucional, tal como o do Estado corroborar a exclusão de minorias sexuais, mediante o veto à doação de sangue. De outro lado, os movimentos sociais de diretos humanos, a questionar esse modelo estigmatizado, higienista e proselitista, considerando as normas de doação antiquadas e atrasadas, mesmo do ponto de vista biológico (Allegretti, 2018).

Hoje em dia, no entanto, existem vários questionamentos biológicos e sociológicos acerca desse "risco" de transmissão, pois o mero fato de praticar sexo anal não é exclusivo de parceiros homoafetivos. Além disso, desconsidera outras características comportamentais que levam a chances distintas de contrair ou não o HIV; como uso de preservativos, número de parceiros sexuais nos últimos 12 meses, testagem recorrente para Infecções Sexualmente Transmissíveis - ISTs, de pessoas que se identificam como HSH e, mais recentemente, a utilização do Profilaxia Pré-Exposição (Prep) e Profilaxia PósExposição (Pep) que são coquetéis antivirais que diminuem em 99\% a chance de pessoas que realizam sexo sem preservativo de contraírem o HIV (Allegretti, 2018).

Independente da sexualidade do indivíduo, o sangue doado sempre é devidamente testado e caso haja contaminação confirmada em exames laboratoriais de pessoas, quer sejam heterossexuais ou não, o sangue será descartado, evitando, assim, seu uso indevido aos pacientes dependentes de hemoderivados. Mesmo assim, a regra até pouco tempo vigente no Brasil, ou seja, até 2020, era a vedação de doação de sangue pela comunidade LGBTQIA+. Caso o candidato afirmasse ter praticado sexo nos últimos 12 meses, ainda que argumentasse que tem parceiro fixo e/ou dissesse fazer de camisinha em suas relações íntimas, a doação era vedada (Aragusuku, 2018).

A política nacional de doação de sangue, por parte de minorias sexuais tem sido discutida por não se apresentar como razoável, além de ser considerada antiquada aos padrões científicos de controle, estocagem e distribuição de sangue na modernidade. Os movimentos sociais de direitos humanos LGBTQIA+, nesse sentido, têm lutado para que a população excluída do direito a doar sangue possa ser incluída no rol da Agencia Nacional de Vigilância Sanitária (ANVISA). No dia 8 de maio de 2020, o Supremo Tribunal Federal (STF) derrubou a restrição que proibia homossexuais de doarem sangue. A votação considerou discriminatórias as regras da ANVISA e do Ministério de Saúde, que vetavam o ato, tornando-as inconstitucionais (Benevides, 2021).

Embora não haja uma previsão expressa que impeça diretamente homens gays ou transgêneros de doarem sangue em razão de sua orientação sexual ou identidade de gênero, a necessidade de abstinência de qualquer prática sexual com outros homens por um longo período gera uma exclusão de fato. O presente trabalho tem por objetivo analisar a Política Nacional de Doação de Sangue no que diz respeito à população LGBTQIA+ considerando em especial o perfil de doadores de sangue em relação a comportamento de risco de contrair Infecções Sexualmente Transmissíveis quanto ao gênero e a sexualidade (Allegretti, 2018).

\section{Metodologia}

O estudo apresentado é do tipo quantitativo e qualitativo, transversal, descritivo e documental. Para tanto, foram analisados documentos relativos às políticas implementadas pela Coordenação-Geral de Sangue e Hemoderivados, do Ministério da Saúde, que coordena o Sistema Nacional de Sangue, Componentes e Derivados (SINASAN) e as normas esparsas que regulamenta a Política Nacional de Sangue, Componentes e Hemoderivados, em especial o decreto no 3.990 , de 30/10/2001, a chamada "Lei do Sangue". O estudo sobre a Política Nacional de Doação de Sangue no Brasil teve por bases os trabalhos publicados e disponibilizados no Google Acadêmico, entre os anos de 1970 a 2020. Utilizando os descritores: 
Política Nacional de Doação de Sangue, Doação de Sangue, LGBTQ e HIV. Foram encontrados um total de 66.899 trabalhos publicados, dos quais foram selecionados apenas 90 artigos científicos. Os critérios de inclusão foram artigos científicos em língua portuguesa, trabalhos completos publicados e com um maior número de citações. Para exclusão foram: artigos repetidos, trabalhos incompletos, língua estrangeira e comunicações breves.

Os artigos foram selecionados a partir da utilização dos descritores e submetidos a três fases de análise, a saber: Fase 1 - leitura dos títulos dos estudos encontrados e exclusão dos que não se enquadraram em qualquer um dos critérios de inclusão deste estudo e dos trabalhos em duplicata; Fase 2 - leitura dos resumos dos estudos selecionados na etapa 1 e exclusão daqueles que, nessa leitura, mostraram-se inadequados aos critérios de inclusão e Fase 3 - leitura na íntegra de todos os estudos préselecionados nas etapas anteriores e triagem final dos trabalhos que tenham maior pertinência e relevância dentro da temática proposta, os quais foram submetidos ao processo de fichamento. Para essa triagem foi elaborada uma grade de análise fechada com os seguintes tópicos: título, autor, ano, objetivo, veículo, $\mathrm{n}^{\circ}$ de páginas, palavras-chave, resumo, conclusão e tipo de pesquisa.

Para aprofundar o assunto, adicionalmente, aplicou-se um questionário por meio de um formulário eletrônico Google Forms ${ }^{\circledR}$, o qual ficou disponível para os respondentes durante 04 (quatro) meses, após a aprovação do Comitê de Ética em Pesquisa com Seres Humanos da Universidade Estadual do Ceará - UECE (Protocolo de aprovação $n^{\circ}$ 3.797.980). O questionário foi direcionado ao grupo "LGBTQIA+ Resistência pela Democracia", no Facebook, composto, por minorias sexuais e simpatizantes da causa LGBTQIA+. Os convites foram enviados para que os participantes, em post aberto no grupo, para que respondessem de forma espontânea, após concordarem (SIM) com Termo de Consentimento Livre e Esclarecido.

As questões que diziam respeito ao comportamento sexual e doação de sangue. Os que responderam o questionário num total de 124, portanto, compuseram a amostra determinada de forma não probabilística, aleatória, intencional e de conveniência. Os critérios de inclusão para participação foram: a) indivíduos que concordem em ler e confirmar/assinar o TCLE e participem da pesquisa; b) adultos maiores de 18 anos e menores de 59 anos; c) indivíduos capazes e alfabetizados. Os dados do questionário foram tabulados com auxílio do programa Excel Microsoft e posteriormente, os resultados foram analisados, por meio do programa IBM SPSS Statistics (versão 20.0; IBM Cop., Armonk, NY, USA) freeware.

\section{Resultados e Discussão}

Dos 90 artigos selecionados, 06 não descreveram o delineamento do estudo, em especial os trabalhos mais antigos. Dos 84 trabalhos restantes, 25 podem ser denominados de pesquisa clínica, seguidas de 20 trabalhos de revisão de literatura e 13 com estudos documentais. Estes 03 tipos de estudos, portanto, correspondem a mais de 50\% de todos os selecionados. $\mathrm{Na}$ maior parte dos trabalhos são focados em estudos na população brasileira, sendo alguns voltados a doenças de interesse à doação de sangue (ISTs) e outros vinculados a discussão e problematização do tema (doação de sangue) para minorias (mulheres, índios, LGBTQIA+, etc). Por conta desses trabalhos terem sido elaborados em diferentes momentos, de 1970 a 2020, os protocolos descritos frequentemente nos artigos antigos quanto ao processo de eleição dos pacientes doadores de sangue eram mais arcaicos, com viés de preconceito social mais rígido. Muito embora os protocolos para aceitação de sangue LGBTQIA+ tenham mudados de fato, recentemente no Brasil, em 2020 (Allegretti, 2018).

No estudo de Milagres (2020), o processo de doação de sangue é colocado um forte caráter normalizador, através de suas técnicas disciplinares: vigilância hierárquica, sanção normalizadora e exame. O poder apresenta-se nos instrumentos utilizados e aparece de forma velada e latente na questão da doação de sangue, colocando padrões considerados como satisfatórios para o perfil do doador de sangue. Com os movimentos sociais de lésbicas, gays, bissexuais, travestis, homens e mulheres transexuais foram se consolidando os direitos humanos das minorias não-heterossexuais nas disputas públicas. Também aconteceram diversas transformações socioculturais, em meio à luta pela construção de uma imagem mais positiva da 
homossexualidade em tempos pós-epidemia de AIDS. A consolidação e expansão sociocultural do chamado "mercado GLS", o início da organização das paradas do orgulho gay nos grandes centros urbanos e a maior visibilidade das populações LGBT nos meios de comunicação (Aragusuku, 2018).

A captação de doadores de sangue é crítica nos hemocentros do país. Excluir parcela da sociedade sem justificativa razoável leva a perda de inestimável de estoques em potencial de sangue, que seriam direcionados aos pacientes necessitados. Estudar as causas e consequências dessa política de triagem de doadores de sangue poderá impactar no processo de desburocratização da captação de hemoderivados. Na questão do poder, Foucault (2014), descreve que a disciplina normaliza diferentes sociedades, em diferentes períodos e contextos incorporando os indivíduos às normas, em função dos objetivos determinados, ou ainda estabelecendo métodos de controle e assujeitamento, segregando aqueles que serão considerados incapazes, daqueles que se encaixam em critérios institucionais estabelecidos para diversos fins.

As nossas formas de ver, agir e sentir, restringem-se e configuram-se ao nosso olhar. Assim, a forma de definir como e o que é a vida estariam determinando ou não a vida ou o sangue de alguém, enquadrados em normas institucionais, sendo políticas, jurídicas e outras (Butler, 2018). No último levantamento realizado no Brasil, foi observado que o percentual de doadores de sangue na população é de apenas 1,8\%, sendo menor do que é preconizado pela OMS. Com isso, o Ministério da Saúde abriu uma campanha para ampliar o número de doadores, com o tema: Fortalecimento da Promoção da Doação Voluntária. Essa Campanha Nacional de Doação Voluntária de Sangue está veiculada a campanha "torcedor sangue bom", desde 2013, teve o objetivo de aumentar o número de doações de sangue por meio do estímulo a novas doações e da fidelização dos doadores regulares. Sua veiculação se dá pelos principais meios de comunicação, inclusive nas mídias sociais; Twitter, Facebook, Instagram (Allegretti, 2018).

De acordo com Foucault (2014), o poder está definido através de protocolos que, a todo momento, reconfiguram as relações que se estabelecem entre os diversos atores envolvidos na doação de sangue, por meio das forças constituídas, produzindo efeitos a partir da manifestação de conhecimentos, experiências e práticas. Para a exclusão da população LGBTQIA+, tínhamos a Portaria 158/2016, cujo Art. 64 considerava-se inapto temporário por 12 (doze) meses homens que tiveram relações sexuais com outros homens e/ou as parceiras sexuais destes. No entanto, em 2020, durante a pandemia do novo coronavírus (COVID-19), os hemocentros do Brasil, apresentaram baixa estocagem de sangue, o que ensejou a liberação da doação de sangue da comunidade LGBTQIA+.

O pesquisador Carpinelli (2016) discutiu a restrição à doação de sangue por 12 (doze) meses para homens que tenham feito sexo com outros homens (HSH), que consta do art. 64 da Portaria n ${ }^{\circ} 2.712$ de 12 de novembro de 2013 do Ministério da Saúde da República Federativa do Brasil. Ele analisou que à luz dos princípios da igualdade e da proporcionalidade, levandose em consideração o conflito de direitos fundamentais que encerra a questão, nomeadamente o direito à saúde e direito à igualdade de tratamento. Foi posta em risco a saúde pública por um hipotético incremento no risco de transmissão de doenças venéreas (sobretudo o HIV) por transfusão sanguínea. Entenderam ser necessário criar um critério que não reforce o estigma social que vivem esses homens levados à condição de marginalidade social e/ou a privação do livre desenvolvimento de suas personalidades, considerando que, trata-se, também, do direito ao igual tratamento que têm esses cidadãos.

O estigma e a discriminação associados ao HIV/Aids, que são relatados pelos usuários, nos serviços da saúde, são também reconhecidos pelos profissionais da saúde como frutos de preconceito, o qual, como mencionado anteriormente está naturalizado pela história social; pela forma como as pessoas com HIV eram rotuladas como integrantes de grupo de risco em face da orientação sexual ou do comportamento de risco. O preconceito, todavia, continua presente na sociedade de modo geral e entre profissionais da saúde, de modo específicos. Os vários relatos de recusa ao tratamento no momento da revelação da doença, dão conta da filiação ideológica presente na fragmentação da assistência, na normatização da demanda com foco em agendamentos e nas estigmatização e desresponsabilização do cuidado. Tudo opera no sentido de dificuldade a realização da 
integralidade dos serviços de saúde para os usuários sem que seja enfatizada a descentralização da oferta do tratamento dos infectados pelo HIV (Guedes, 2020).

Em relação a sexualidade, Giddens (1993) aborda a questão da intimidade na modernidade tardia ocidental. Reflete como parâmetro para análise e compreensão das mudanças da intimidade, ressaltando o papel da mulher como agente catalisador das novas configurações interacionais de cunho socioafetivo que se estabelecem frente às transformações da intimidade. Mostra o quando a sexualidade tem tido papel central na construção da nossa sociedade e também o estigma acerca do homoerotismo sempre visto como contato íntimo reprimido pelas normas ocidentais. Ressalta o papel dos movimentos sociais na transformação das subjetividades trazendo esse conceito ao tratamento de homoafetivos em relação a doação de sangue podemos também ver o quanto a sexualidade diversa da "norma", por isso, quer se fale de heterossexualidade quer que se fale da homossexualidade, o que diverge de forma social pode ser estigmatizado o que contribuir para o preconceito do "sangue gay" nos hemocentros.

As políticas de enfrentamento de HIV/AIDS deram visibilidade a grupos e populações estigmatizadas, como os homossexuais, as trabalhadoras do sexo e as pessoas trans, que permaneceram esquadrinhandos nos chamados "grupos de risco". Com isso, pessoas ligadas a movimentos capazes de produzir solidariedade e formas de sociabilidade da ordem das resistências, iniciaram ações voltadas para as pessoas afetadas pelo vírus e pela doença, as quais favoreceram o fortalecimento do movimento homossexual no Brasil. Por meio de associações e grupos gays, foram estabelecidas relações de duplo condicionamento entre estratégias locais de cuidado de si e estratégias globais de governo biopolítico (Baturi Junior; LARA, 2018).

Michel Foucault (2014), há muito evidencia que na sociedade está instalado culturalmente o silêncio no que se refere à sexualidade, gerando repressão. Esse sentimento de repressão condena as práticas corpóreas fogem à normatividade devido ao status vigente e que devem ser seguidos para que o indivíduo seja aceito na sociedade. Essas ações tornam a sexualidade silenciadas e as práticas ligadas ao prazer condenada ao ostracismo. $\mathrm{O}$ ato de expor as diferentes homossexualidades e reafirmá-las torna-se um ato político e também uma tomada de decisão diante de normas sociais que reprimem essas expressões. Dessa forma, as identidades da comunidade LGBTQIA+ se constroem com fruto de lutas políticas de movimentos cuja missão é de construir ambientes de acolhimento e pertencimento, para que a vivência das pessoas e suas homossexualidades possam ser defendidas como legítimas.

Os movimentos LGBTs no Brasil tornou-se mais visível por meio da organização de manifestações de rua, conhecidas como "Paradas do Orgulho LGBT", nas quais milhares de pessoas passaram a se unir uma vez por ano, em várias cidades do país. Críticas severas têm sido feitas às paradas, considerando que são manifestações públicas em excesso, que se aproximam de um carnaval de fora de época e que não conseguem se transformar em ações políticas concretas, tais como novas propostas de leis de combate a homofobia ou eleição de políticos LGBTs para a representação parlamentar (Mello, 2012). Apesar dessas críticas, recentemente, foi aprovada Lei contra a homofobia e também, eleitos alguns representantes pertencentes às minorias LBGTQIA+ para os parlamentos municipais, estaduais e federal.

Outro desafio na construção de políticas LGBT no Brasil é o de que, no interior dessas políticas, estaria o agenciamento de uma mudança cultural, crenças, valores e no imaginário social. As políticas de gênero e sexualidade reduzem o risco de os sujeitos assimilarem modelos hegemônicos de lesbo-homo-bi-transexualidade, reforçando pelo pensamento conservador brasileiro, no qual a Igreja exerce grande influência (Feitosa, 2019). A atuação política dos coletivos LGBT abriu caminhos para o desenvolvimento de estratégias de superação de preconceitos e estigmas que têm por base gênero, para homens e mulheres homoeroticamente orientados pudessem construir uma nova cidadania, inclusiva e multicultural, por meio do resgate da participação em espaços sociais nos quais eram destruídos inclusive da palavra e da expressão de suas demandas (Silva, 2012). 
Após a Doação de Sangue pela População LGBTQIA+ ter sido autorizada no Brasil pelo Supremo Tribunal Federal a $137^{\circ}$ Promotoria de Justiça do Estado do Ceará - Defesa de Saúde Pública encaminhou à Secretaria de Saúde do Estado do Ceará, ao HEMOCE e à Fujisan a Recomendação no 0025/2020/137ªPmJFORm pugnando pela aceitação imediata de doações de sangue feitas por as pessoas LGBTQIA+, sem discriminação em razão de sua orientação sexual ou identidade de gênero, conforme na ADI $n^{\circ} 5.543$ do STF. Além disso, solicitou novos protocolos para doação de sangue e que a sociedade fosse informada por meio das redes oficiais de comunicação, sobre a possibilidade de doação de sangue pelas pessoas LGBTQIA+.

Apesar dos avanços sociais e jurídicos para superar preconceitos e estigmas que recaem sobre a doação de sangue feita pela comunidade LGBTQIA+, há, ainda, muito receio para o acolhimento e a aceitação do sangue de indivíduos que vivem abertamente sua sexualidade e conjugam suas identidades de gênero diversa da heteronormatividade. Em pesquisa recente, desenvolvida pelo autor deste estudo, ficou evidente que preconceitos e estigmas que estão em larga medida presentes na população em geral não espelham, de fato, a realidade concreta dos doadores de sangue pertencentes à comunidade LGBTQIA+ quanto aos cuidados com a saúde e comportamento sexual de risco.

A amostra foi composta por 124 participantes, em sua maioria apresentando ensino superior completo e pós graduação (24,2\% e 55,6\%, respectivamente). Em 42,7\% dos indivíduos relataram ter feito alguma doação de sangue na vida.Acerca da orientação sexual, a maioria dos casos se referiram como heterossexuais (46\%) e homossexuais $(45,2 \%)$, assim como a maioria adotaram a identidade de gênero do tipo cisgênero $(84,7 \%)$. Em relação aos relacionamentos, 65,3\% tinham um parceiro fixo e apresentou associação estatisticamente significativa em relação a ser doador de sangue ( $n=41, p=0,048)$, Tabela 2. Em apenas 1 caso relatou ser profissional do sexo e não era doador de sangue. Em $58,9 \%$ da amostra foi testada alguma vez na vida para o HIV tanto nos doadores quanto nos não doadores.

Nos hemocentros, cerca de 33,0\% foram questionados sobre sua orientação sexual e/ou identidade de gênero apresentando associação estatisticamente significativa $(\mathrm{p}<0,001)$ para a realização desta pergunta nesses centros. Em 40,3\% não tiveram impedimentos para realizar a doação, mas em $8 \%$ foram impedidos e em 5,7\% algumas vezes foram impedidos de doar devido a sua orientação sexual e/ou identidade de gênero, apresentando associação estatisticamente significativa (p $<0,001$ ), Tabela 1.Em 95,2\% dos participantes não concordam com o impedimento de doação de sangue em pacientes LGBTQIA+ sem nenhum teste prévio realizado e em 93,5\% tem conhecimento de que testes prévios são realizados no sangue do indivíduo para detecção do HIV, além disso, em 92,7\% dos indivíduos relataram que não estariam correndo risco se recebesse sangue de grupos LGBTQIA+ do que heterossexuais, tais dados não apresentaram associação significativa em relação a doação de sangue (Tabela 1). 
Tabela 1. Associação entre doadores de sangue e opiniões respondidas no questionário sobre orientação sexual.

\begin{tabular}{|c|c|c|c|}
\hline \multirow{2}{*}{$\begin{array}{ll}\text { Escolaridade } & \text { Questionário } \\
\end{array}$} & \multicolumn{2}{|c|}{ Doadores } & \multirow[b]{2}{*}{$p$} \\
\hline & Sim & Não & \\
\hline Ensino Médio Incompleto & 0 & 2 & $0,432^{\mathrm{a}}$ \\
\hline Ensino Médio Completo & 2 & 3 & \\
\hline Superior Incompleto & 6 & 12 & \\
\hline Superior Completo & 11 & 19 & \\
\hline Pós-Graduado & 34 & 35 & \\
\hline \multicolumn{4}{|l|}{ Orientação sexual } \\
\hline Heterossexual & 28 & 29 & $0,327^{\mathrm{a}}$ \\
\hline Homossexual & 20 & 36 & \\
\hline Bissexual & 4 & 6 & \\
\hline Prefiro_não_responder & 1 & 0 & \\
\hline \multicolumn{4}{|l|}{ Identidade de gênero } \\
\hline Cisgênero & 48 & 57 & $0,393^{\mathrm{a}}$ \\
\hline Transgênero & 1 & 1 & \\
\hline Nâo_binário & 1 & 3 & \\
\hline Prefiro_não_responder & 3 & 10 & \\
\hline \multicolumn{4}{|l|}{ Parceiro fixo } \\
\hline Sim & 41 & 40 & $0,048^{\mathrm{a} *}$ \\
\hline Não & 11 & 27 & \\
\hline Sim,porém não há fidelidade conjugal & 1 & 4 & \\
\hline \multicolumn{4}{|l|}{ Profissional do sexo } \\
\hline Sim & 0 & 1 & $1,000^{\mathrm{b}}$ \\
\hline Não & 53 & 70 & \\
\hline \multicolumn{4}{|l|}{ Uso do preservativo } \\
\hline Sim & 19 & 28 & $0,746^{\mathrm{a}}$ \\
\hline Não & 22 & 23 & \\
\hline Somente se fizer fora do relacionamento & 6 & 11 & \\
\hline Algumas vezes & 6 & 9 & \\
\hline \multicolumn{4}{|l|}{ Testado (a) para o HIV? } \\
\hline Sim & 35 & 38 & $0,161^{\mathrm{a}}$ \\
\hline Não & 18 & 33 & \\
\hline \multicolumn{4}{|c|}{ Nos hemocentros, lhe perguntaram sobre sua orientação sexual e/ou identidade de gênero? } \\
\hline Sim & 27 & 14 & $<0,001^{\mathrm{a} *}$ \\
\hline Não & 16 & 9 & \\
\hline As vezes, mas nem sempre & 8 & 0 & \\
\hline Nunca participei de doação de sangue & 2 & 48 & \\
\hline \multicolumn{4}{|c|}{ Já foi impedido (a) de doar sangue por conta de sua orientação sexual? } \\
\hline Sim, nunca pude doar sangue & 3 & 7 & $<0,001^{a *}$ \\
\hline Não, nunca fui impedido de doar sangue & 42 & 8 & \\
\hline Algumas vezes sim, mas já doei sangue & 7 & 0 & \\
\hline Nunca tentei doar sangue & 1 & 56 & \\
\hline \multicolumn{4}{|c|}{ Você acha correto impedir que LGBTQIA+ de doarem sangue? } \\
\hline Sim & 2 & 4 & $1,000^{\mathrm{b}}$ \\
\hline não & 51 & 67 & \\
\hline \multicolumn{4}{|c|}{$\begin{array}{l}\text { Você tem conhecimento de que independente da orientação sexual da pessoa, o sangue é } \\
\text { testado? }\end{array}$} \\
\hline Sim & 51 & 65 & $0,465^{\mathrm{b}}$ \\
\hline Não & 2 & 6 & \\
\hline \multicolumn{4}{|c|}{$\begin{array}{l}\text { Caso você necessitasse receber sangue, e a origem fosse de um doador LGBTQIA+ mesmo } \\
\text { sendo testado acharia que estaria correndo algum risco? }\end{array}$} \\
\hline Sim & 4 & 5 & $1,000^{\mathrm{b}}$ \\
\hline Não & 49 & 66 & \\
\hline
\end{tabular}


O uso de preservativo esteve presente em 37,9\% da amostra e verificou-se uma tendência ao não uso de preservativo quando o relacionamento ocorria com o parceiro fixo $(n=44, p<0,001)$, Tabela 2. Quanto a orientação sexual, os homossexuais foram os grupos que mais faziam o uso do preservativo e em relação a identidade de gênero, o cisgênero fez maior uso do preservativo, o grupo transgênero o que não usou preservativo tinha parceiro fixo e o grupo binário referiu fazer o uso somente nas relações sexuais fora do relacionamento, tais dados não apresentaram associação significativa.

Tabela 2. Associação entre o uso de preservativo e orientação sexual e/ou identidade de gênero e parceiro fixo.

\begin{tabular}{lllllc}
\hline & \multicolumn{7}{c}{ Uso de preservativo } \\
\hline Orientação sexual & Sim & Não & Somente fora & Algumas vezes & $\boldsymbol{p}$ \\
Heterossexual & 19 & 24 & 9 & 5 & $0,455^{\mathrm{a}}$ \\
Homossexual & 20 & 20 & 7 & 9 & \\
Bissexual & 7 & 1 & 1 & 1 & \\
Prefiro_não_responder & 1 & 0 & 0 & 0 & $0,360^{\mathrm{a}}$ \\
Identidade de gênero & & & & 13 & \\
Cisgênero & 40 & 37 & 15 & 0 & \\
Transgênero & 1 & 1 & 0 & 1 & $<0.001^{\mathrm{a} *}$ \\
Nâo_binário & 1 & 0 & 2 & & \\
Prefiro_não_responder & 5 & 7 & 0 & 7 & 8 \\
Parceiro fixo & & & & 0 & \\
$\quad$ Sim & 19 & 44 & 11 & \\
Não & 27 & 1 & 2 & & \\
Sim, porém não há fidelidade conjugal & 1 & 0 & 4 & & \\
\hline
\end{tabular}

Fonte: Autores.

Em relação aos relacionamentos, 65\% afirmou ter um parceiro fixo, o que apresentou associação estatisticamente significativa em relação a ser doador de sangue $(n=41, p=0,048)$. Apenas uma pessoa relatou ser profissional do sexo e não era doador de sangue. Cerca de 33,0\% informaram que foram questionados sobre sua orientação sexual e/ou identidade de gênero nos hemocentros, o que apresentou associação estatisticamente significativa $(p<0,001)$ para a realização desta pergunta nesses centros. $40,3 \%$ não tiveram impedimentos para realizar a doação, mas $8 \%$ foram impedidos e $5,7 \%$ algumas vezes foram impedidos de doar devido a sua orientação sexual e/ou identidade de gênero, apresentando associação estatisticamente significativa $(p<0,001)$.

Quase a totalidade da amostra, isto é, demostrou que 95,2\% dos participantes não concordou com o impedimento de doação de sangue de doadores LGBTQIA+ sem nenhum teste prévio realizado e em 93,5\% demonstraram ter conhecimento de que testes prévios são realizados no sangue do indivíduo para detecção do HIV, enquanto 92,7\% dos indivíduos relataram que não estariam correndo risco se recebesse sangue de grupos LGBTQIA+.A julgar pelo uso do preservativos, parece evidente que o processo de avaliação da elegibilidade de doadores deve ser rigoroso, porque essencial à proteção da saúde tanto dos destinatários da transfusão quanto dos próprios doadores de sangue. Ademais, tal processo reduziria significativamente o tempo em que elegíveis precisariam adiar doações em função de terem ou não mantido relações sexuais.

O adiamento de 3 meses é apoiado por evidências de grupos considerados de alto risco, como homens que fazem sexo com homens, porém tais grupos permanecem parcialmente excluídos. Uma solução para isso pode ser a introdução de uma avaliação individual de comportamento de risco, a qual é cega para a orientação sexual, como tem sido implementado em vários países, incluindo Espanha e Itália (Offergeld, 2014). Quanto aos comportamentos sexuais, vem se observando um aumento no uso de preservativos entre os homossexuais e o seu uso é menor em populações mais velhas e com menor acesso aos sistemas de saúde. Além disso, homens que praticam sexo anal receptivo, os chamados "passivos" e ambos sexo anal 
insertivo e receptivo, os chamados "versáteis" correm maior risco de Infecção por HIV do que homens que praticam apenas sexo anal insertivo, os chamados "ativos (Allegretti, 2018).

Parece estar claro que a exclusão de LGBTQIA+ da doação de sangue está perpassada pelo preconceito institucional histórico e não pelo risco em potencial. Os argumentos médicos de janela imunológica e prevalência do vírus em população LGBTQIA+ não se sustentam, pois apesar de haver maior prevalência do vírus HIV nesta população, não há como afirmar que há mais segurança entre os indivíduos que tem parceiro fixo e usam preservativo. Tampouco não há como saber com fidedignidade se os indivíduos respondem a anamnese de triagem com total veracidade dos fatos (Allegretti, 2018). Independentemente do tipo de relação sexual, qualquer pessoa que faz sexo (ou seja, toda a população) poderia, ser portadora em potencial do HIV e nem por isso as doações deixariam de ocorrer, uma vez que, como já salientado, testes altamente seguros são realizados no sangue dos doadores.Seja como for a aceitação verificada entre os participantes da pesquisa que empreendemos em relação ao recebimento de transfusões sanguíneas da comunidade LGBTQIA+ e o conhecimento acerca dos procedimentos, de realização de testes prévios para descartar a possibilidade de transmissão das ISTs são contrários ao impedimento dos hemocentros para o recebimento de sangue dessa comunidade.

\section{Conclusão}

A Política Nacional de Doação de Sangue reflete a forma como a sociedade se posiciona diante de determinadas questões, demonstrando que os valores adquiridos no convívio social são bases para a discriminação que a parcela LGBTQIA+ brasileira enfrenta na doação de sangue. Todavia, tal discriminação configura-se como elemento prejudicial ao avanço da saúde no país, porquanto impede que milhares de litros de sangue sejam doados. Nesse âmbito, pode-se analisar que essa problemática é sustentada, sobretudo pelo preconceito histórico da sociedade e pelas limitações impostas pela legislação federal. Ao analisar a evolução dessa política pública, a priori, comumente, as instituições ligam as ISTs, como HIV, aos LGBTQIA+, uma vez que, por não haver o risco de engravidar, julga que a maioria não usa preservativos nas relações sexuais. Tendo em vista esse estereótipo, o preconceito torna-se enraizado na sociedade. Por conseguinte, minorias sexuais, regularmente, acabam sendo impedidas de doar sangue, sem que haja testes prévios para deteç̧ão de ISTs pelo simples fato de responder na anamnese de triagem de doadores que não é heterossexual e/ou cisgênero.

Podemos observar a necessidade de criação de uma nova lente para a sociedade brasileira enxergar a doação de sangue pelas minorias sexuais. Soma-se a isso, é papel do governo, por meio de leis, de instituir a obrigatoriedade da realização de testes de detecção de ISTs mais sofisticadas como o NAT, a fim de que as oportunidades de doação sejam democratizadas, sem estigmas apesar sobre a sexualidade e/ou identidade de gênero. Quando analisamos os questionários da pesquisa que realizamos ficou evidente que muitos LGBTQIA+ foram impedidos de doar sangue ou, para conseguir, tiveram que omitir sua condição sexual para não serem barrados. Isso demonstra a total desigualdade de oportunidades de participar do processo de doação do grupo LGBTQIA+ quando comparado à população em geral.

\section{Referências}

Allegretti, A. C. V. (2018). Redes sociais na produção científica em administração pública da saúde no brasil. Rev. Adm. Pública.

Aragusuku, H. A. (2018). Políticas públicas e cidadania LGBT em Mato Grosso: Uma década de avanços e retrocessos (2007-2017). Sex., Salud Soc. Rio de Janeiro.

Benevides, M. G. (2021). Direitos Humanos de Gênero: produção e reprodução de saberes. Ciências Sociais Contemporâneas: Objetos de Pesquisa. EDUC.

Butler, J. (2018). Quadros de guerra. Civilização Brasileira.

Carpinelli, A. P. T. (2016). Doação de sangue por homens que fazem sexo com outros homens à luz do princípio da igualdade no direito brasileiro. Iuris in mente: revista de direito fundamentais e políticas públicas, 1(1):10. 
Research, Society and Development, v. 10, n. 10, e517101019306, 2021

(CC BY 4.0) | ISSN 2525-3409 | DOI: http://dx.doi.org/10.33448/rsd-v10i10.19306

Foucault, M. (2014). Microfisica do poder. (28a ed.), Paz \& Terra.

Feitosa, C. (2019). Políticas públicas LGBT no Brasil: um estudo sobre o Centro Estadual de Combate à Homofobia de Pernambuco. Sexualidad, Salud y Sociedad.

Guedes, H. C. S. (2020). Integralidade na Atenção Primária: análise do discurso acerca da organização da oferta do teste rápido anti-HIV. Escola Anna Nery. Rio de Janeiro.

Giddens, A. (1993). New Rules of Sociological Method (2a ed.). Stanford University Press.

Mello, L. (2012). Questões LGBT em debate: sobre desafios e conquistas. Soc. e Cult, 15(1): 151-161.

Milagres, S. V. (2020). Expressão das relações de poder na conformação das práticas de doação de sangue. Dissertação (Mestrado): Universidade Federal de Minas Gerais.

Offergeld, R. (2014). Sexual risk behaviour and donor deferral in Europe. Vox Sang, 107(4): 420-427.

Silva, A. S. (2012). Memória, Consciência e Políticas Públicas: as Paradas do Orgulho LGBT e a construção de políticas públicas inclusivas. Revista Electrónica de Psicologia Política.

Vianna, C. P. (2015). O movimento LGBT e as políticas de educação de gênero e diversidade sexual: perdas, ganhos e desafios. Educação e Pesquisa. 